\title{
Reformation process of the neuronal template for nestmate-recognition cues in the carpenter ant Camponotus floridanus
}

\author{
Sara Diana Leonhardt • Andreas Simon Brandstaetter • \\ Christoph Johannes Kleineidam
}

\begin{abstract}
Ants use cuticular hydrocarbons (CHC-profiles) as multicomponent recognition cues to identify colony members (nestmates). Recognition cues (label) are thought to be perceived during ant-ant encounters and compared to a neuronal template that represents the colony label. Over time, the CHC-profile may change, and the template is adjusted accordingly. A phenotype mismatch between label and template, as happens with CHC-profiles of foreign workers (nonnestmates), frequently leads to aggressive behavior. We investigated the template reformation in workers of the carpenter ant Camponotus floridanus by masking their antennae with postpharyngeal gland (PPG) extracts from nestmates or non-nestmates. The behavioral response of manipulated workers encountering unmanipulated workers was measured independently after 2 and after $15 \mathrm{~h}$. After $2 \mathrm{~h}$ of incubation, workers treated with either of the two PPG-extracts showed low aggression towards nestmates and high aggression towards non-nestmates. In contrast, after $15 \mathrm{~h}$ of incubation, workers treated with non-nestmate PPG-extract showed low aggression towards both nestmates and non-nestmates. The slow ( $>2 \mathrm{~h}$ ) adjustment of the template indicates a reformation localized in the central nervous system rather than in chemosensory neurons. In addition, our data show that template adjustment to a new CHC-profile does not impair the assessment of the old CHC-profile as nestmate label.
\end{abstract}

Keywords Postpharyngeal gland · Cuticular hydrocarbons · Chemical communication · Hymenoptera . Aggressive behavior

S. D. Leonhardt · A. S. Brandstaetter · C. J. Kleineidam $(\bowtie)$ Department of Sociobiology and Behavioral Physiology, Biozentrum, University of Würzburg, Am Hubland, 97074 Würzburg, Germany

e-mail: kleineidam@biozentrum.uni-wuerzburg.de

\section{Introduction}

In social insects, colony survival depends on an elaborate chemical system which ensures identification of conspecifics, nestmates, and kin (Hölldobler and Wilson 1990; Hölldobler 1995). Particularly, cuticular hydrocarbons (CHCs) have been found to serve as chemical recognition cues in ants (Howard 1993; Singer 1998; Lahav et al. 1999; Lucas et al. 2005). The primary function of CHCs is to preserve insects from desiccation, cuticle abrasion, and infection, unless they became further involved in the social recognition system of colony-building insects (Lockey 1988). Across colonies of the same species, CHC-profiles consist of the same components, although in different ratios (Vander Meer and Morel 1998). Within a colony, CHC-profiles are not stable, but are influenced by environmental factors and vary with age, reproductive status, and/or caste membership of their bearers (Morel et al. 1988; Wagner et al. 1998; Nielsen et al. 1999; Heinze et al. 2002; Dietemann et al. 2003; D'Ettorre et al. 2004; Endler et al. 2004; Buczkowski et al. 2005; Katzerke et al. 2006). The interindividual variation of $\mathrm{CHC}$-profiles requires that the workers' acceptance range for nestmate CHC-profiles is wide enough to account for caste- or age differences. In contrast, the temporal variation of the colony CHC-profile implies that ants permanently adjust and confine their acceptance range to allow a reliable discrimination from non-nestmates.

It is thought that information about the colony-specific CHC-profile is represented as a neuronal template in the nervous system. During encounters with other individuals, recognition cues (label) of the CHC-profile are perceived and compared to the template in a process described as phenotype matching (Crozier and Dix 1979; Lacy and Sherman 1983; Getz and Chapman 1987; Crozier and Pamilo 1996; Hauber and Sherman 2001). A mismatch between label and 
template, as happens with CHC-profiles of non-nestmate workers, generally results in aggressive behavior (Lacy and Sherman 1983). The formation and adjustment of the template requires at least simple forms of learning (e.g. adaptation or habituation) and frequent interaction between colony members to unify the colony label via trophallaxis and allogrooming (Soroker et al. 1994; Dahbi et al. 1999). The postpharyngeal glands (PPGs) play an important role in this unification, and their content shows a strong congruence to the cuticular CHC-profile (Bagneres and Morgan 1991; Soroker et al. 1994). As a pool for inherent and exogenous hydrocarbons, the PPGs are used for distributing $\mathrm{CHCs}$ such that all workers possess the same CHC-profile and share a so-called 'Gestalt colony odor'. The misleading term 'Gestalt' was introduced by Crozier and Dix (1979) in the 'Gestalt model', probably to emphasize the complexity and the emergent properties in the nestmate recognition system. 'Gestalt colony odor' is still used and even led some authors to term the postpharyngeal glands a 'Gestalt organ' for nestmate recognition (Soroker et al. 1994; Lenoir et al. 1999, 2001; Boulay et al. 2004; Akino and Yamaoka 2005). It is important to note that the term 'Gestalt' in Psychology and in Neurobiology describes a phenomenon of perception (von Ehrenfels 1890; Wertheimer 1925; Kandel and Wurtz 1990). Thus, a concept actually associated with the brain has been misleadingly transferred to the chemical properties of the cuticle and the postpharyngeal glands, confusing what is perceived and what is detected. For the sake of consistent terminology, the term 'Gestalt' may be used in the context of nestmate recognition in the same way as in Neurobiology and in Psychology, that is as a description of brain function.

It is not known, where the template is localized in the nervous system. The template may be localized in the central nervous system (CNS). In case the template were localized in the CNS, aggression would be regulated by a threshold of similarity between template and label (Crozier and Dix 1979; Obin and Vander Meer 1989; Vander Meer and Morel 1998). Alternatively, the adaptation of the worker's olfactory receptor neurons (ORNs) due to constant stimulation with its own CHC-profile may function as a template. The latter scenario was recently proposed by Ozaki and colleagues who found that ORNs of one particular chemosensory sensillum were responsive only to $\mathrm{CHC}$ extracts of non-nestmates, but were unresponsive to $\mathrm{CHC}$ extracts of nestmates (Ozaki et al. 2005). Ozaki and colleagues suggested a peripheral recognition mechanism for detection of colony-specific recognition cues.

Depending on the mechanism utilized for template reformation, the adjustment to a changing label is expected to occur at different time scales with peripheral adaptation (ORN-adaptation) occurring faster than plasticity in the central nervous system (CNS-plasticity).
In this study, we investigated the template reformation in workers of the carpenter ant Camponotus floridanus following a manipulation of their sensory apparatus. The sensory experience of workers was manipulated by masking their own CHC-profile on the antenna with $\mathrm{CHCs}$ obtained from PPGs of other (nestmate and non-nestmate) workers. The behavioral response (aggressive behavior) of manipulated workers towards single unmanipulated nestmates or non-nestmates was measured either after $2 \mathrm{~h}$ or after $15 \mathrm{~h}$. The change in behavior was used as a measure for the reformation process of the template, and the time of change for reformation is discussed with respect to the two proposed mechanisms, ORN-adaptation and CNS-plasticity.

\section{Materials and methods}

\section{Animals}

For behavioral experiments with manipulated workers we used a laboratory colony of $C$. floridanus. The founding queen of the colony (colony C316) had been collected by Endler and Diederich at the Sugarloaf Shores, FL, USA, in 2003 and reared in an environmental chamber at $25^{\circ} \mathrm{C}$ and $50 \%$ relative humidity in a $12 \mathrm{~h} / 12 \mathrm{~h}$ photoperiod. At the time of the experiments, the colony had about 4,000 individuals and was provided with honeywater, dead cockroaches (Nauphoeta cinerea), and artificial diet (Bhatkar and Whitcomb 1970), twice a week, and water ad libitum. Only large workers (head with $>3 \mathrm{~mm}$ ) were used for behavioral experiments.

PPG dissection and extract application

The postpharyngeal glands (PPGs) for manipulation of other workers' antennae were obtained from $\mathrm{CO}_{2}$ anesthetized small workers (head with $<3 \mathrm{~mm}$ ) by dissecting their heads. The postpharyngeal glands were transferred to a cleaned microscope slide and carefully squeezed with a tweezers' tip to open the glands and disperse the extract on the slide. Immediately afterwards, an immobilized (on ice) large worker was swayed headfirst above the microscope slide with only its antennae touching the slide surface, so that the PPG extract was evenly applied on its antennae. This procedure lasted no longer than $30 \mathrm{~s}$ because the PPG extract dried up rapidly. We used one gland per large worker of $C$. floridanus. We established two groups of manipulated workers according to different PPG treatments of their antennae. One group of workers was treated with PPG extract from nestmates (colony C316), whereas the other group received a treatment with PPG extract obtained from workers of a foreign colony (non-nestmates; colony C90), the founding queen of which had been collected by 
Endler and Strehl on Orchid Island, FL, USA, in 2001. The non-nestmate colony was similar in size and reared under the same conditions as the nestmate colony.

Behavioral experiments with yoke-workers

Manipulated workers were restrained in a modified Petridish. We cut a slit into the rim of a Petri-dish that was wide enough to let a large worker's neck slip through (approx. $0.5 \mathrm{~mm}$ ). Alike the yoke for oxen, the Petri-dish (yokedish) was overturned and put over the worker's neck (similar to the immobilization device used by Lucas et al. 2005). A piece of adhesive tape was placed above the worker's head to adjust its position, and the body posterior to the neck was free to move inside the yoke-dish (Fig. 1a, c). The yoke prevented the workers from cleaning their antennae. However, the workers restrained in the yoke-dish (yokeworkers) were still mobile enough for behavioral assays. The opening of the mandibles, movements of the antennae, and movements of legs and gaster could be observed which allowed us to describe behavioral responses of yoke-workers more accurately than it is possible with other devices commonly used for behavioral experiments.

Yoke-workers were placed on a wooden board about $25 \mathrm{~cm}$ above a cleaned bench with the workers' heads positioned at the rim of the board. Thus, the workers' mandibles and their manipulated antennae were located in midair.

We observed the behavioral response of yoke-workers in controlled encounters with either nestmates (from colony C316) or non-nestmates (from colony C90) after $2 \mathrm{~h}$ (61 individuals) or after $15 \mathrm{~h}$ (61 individuals). Five large workers were transferred to a wooden bridge of equal height to the board with the yoke-workers $(25 \mathrm{~cm}$ above the bench top). The gap in-between the end of the bridge and the board was small enough to allow antennation, but wide enough to prevent the freely moving workers (henceforth called encounter-workers) from crossing the gap or biting the yoke-workers (Fig. 1c). As soon as one encounterworker from the bridge approached and antennated the yoke-worker, the behavioral response of the yoke-worker was observed during the first $5 \mathrm{~s}$ of an encounter and scored according to the following behavioral index:

- 0: no reaction

- 1: antennation, mandibles closed

- 2: mandibles slightly open

- 3: mandibles widely open

- 4: wide opening and immediate closing of mandibles ("biting")

This index had been established during several pre-testing trials with untreated individuals. We recorded the highest aggression response observed. In order to obtain a more

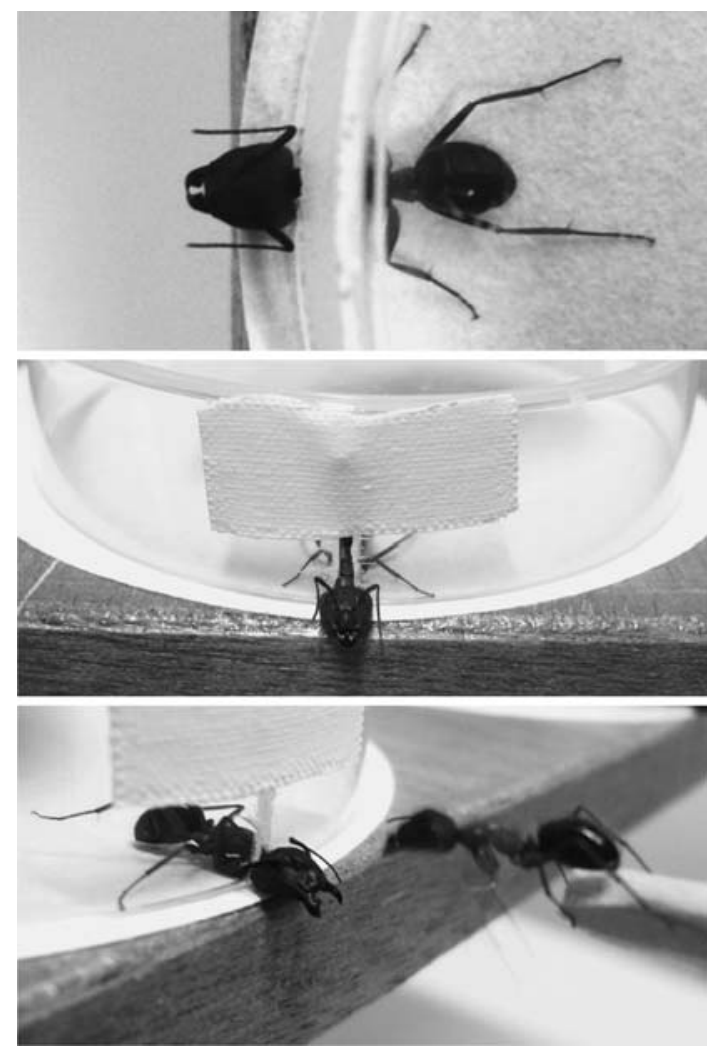

Fig. 1 Yoke-dish with a tethered worker of $C$. floridanus as used in the behavioral experiments. a View from above showing the vertical rim of the Petri dish (yoke-dish, white) which functions as a yoke for the worker. b: Frontal view on the head of a yoke-worker, the neck is fixated by the gap of the yoke-dish. c: Interaction of a freely moving worker (encounter-worker) with a yoke-worker. In the experiments, the behavioral response of both, yoke- and encounter-worker, was recorded during an interaction

accurate description of the workers' behavior, also intermediate scores were used when the behavioral response could not be unambiguously assigned to an integer score.

In addition to the behavioral response of yoke-workers, we further noted the behavior of encounter-workers to count for any bias in the behavioral response of yoke-workers. Because encounter-workers initiated the behavioral sequence of each encounter the behavioral response of yoke-workers may be induced by the behavior shown by the encounter-workers. We noted whether encounter-workers acted aggressively (open mandibles, attempts to attack) or non-aggressively (closed mandibles, sometimes attempts to perform trophallaxis).

Yoke-workers as well as encounter-workers were tested individually and only once. The encounter-workers were gently removed from the bridge after interaction with a yoke-worker. The kind of PPG treatment the yoke-workers had received was balanced on all days of experiments, and the observer was blind to the PPG treatment. The nestmate and non-nestmate encounter-workers (n-NM or NM 
encounter-workers) were presented to the yoke-workers pseudo randomly.

\section{Statistical analysis}

The behavioral responses of yoke-workers was analyzed separately for the groups tested after $2 \mathrm{~h}$ and the groups tested after $15 \mathrm{~h}$, using the Kruskal-Wallis ANOVA (KWtest) followed by multiple comparisons (mc) between groups.

In order to analyze whether the behavior of encounterworkers correlates with the behavior of yoke-workers, we calculated the Spearmans-Rank correlations (SR) for all data pooled and for each group separately. If the behavioral response of yoke-workers depends on the behavior of encounter-workers we expect to find the same differences in behavior in both encounter-workers and yoke-workers. Therefore, we tested the behavior of encounter-workers between independent groups of nestmates and non-nestmates, using the $\mathrm{Chi}^{2}$-test (with Fishers exact $\mathrm{p}$ ), and compared the differences to the differences found in the behavior of yoke-workers.

All calculations were performed using the software Statistica 7.1 (StatSoft, Tulsa, OK, USA).

\section{Results}

Workers restrained in the yoke-device quickly calmed down within 3 to $5 \mathrm{~min}$ and adopted a posture with retreated antennae. Only in response to disturbance, e.g. an encounter with another worker, yoke-workers started moving their antennae and mandibles. We recorded the behavioral response of yoke-workers only for a short period, right after onset of the interaction with encounter-workers, because, later on, the yoke-workers tried to escape the yoke-dish by pulling with their legs and bending their gaster. This behavioral pattern was similar irrespective of the time the yokeworkers were tested. The constancy in behavior indicates that workers restrained in the yoke-device were in good physical condition throughout the experiments.

Yoke-workers responded with low aggression (median aggression score of 2 or less) when encountering a nestmate (NM encounter-workers, Fig. 2a, b; left). The four groups of yoke-workers tested after $2 \mathrm{~h}$ of incubation differed significantly in their behavioral response towards encounterworkers (Fig. 2a, KW-test: $\mathrm{H}(3 ; 62)=9.84 ; P<0.05$ ). However, we found no difference between NM yoke-workers and n-NM yoke-workers, neither in encounters with NM- nor in encounters with n-NM encounter-workers (mc: $P>>0.05$ for both pairs). Irrespective of treatment, yoke-workers responded less aggressively towards NM encounter-workers than towards n-NM encounter workers

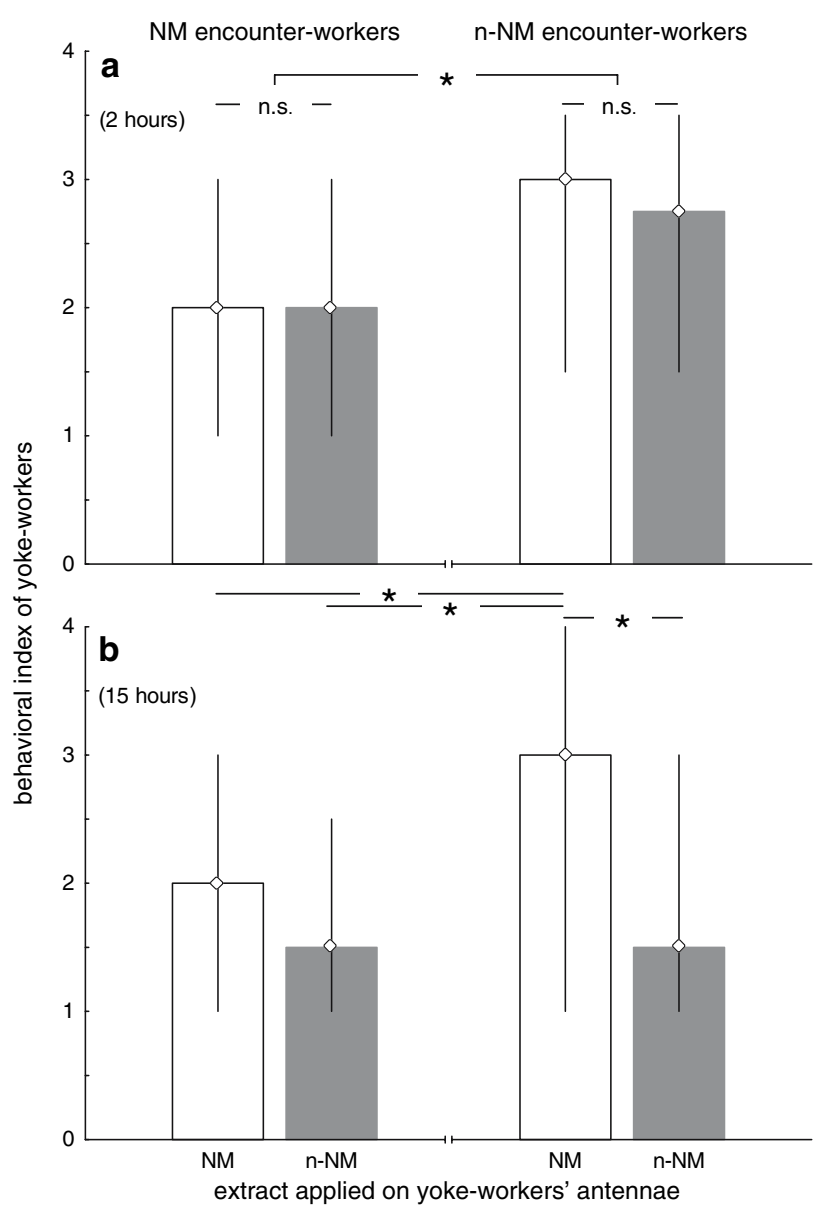

Fig. 2 Behavioral responses of yoke-workers treated with nestmate PPG extract (NM, light bars) and non-nestmate PPG extract (n-NM, dark bars) towards nestmate (left side) and non-nestmate encounterworkers (right side). Yoke-workers were tested either after $2 \mathrm{~h}$ (a) or $15 \mathrm{~h}$ (b) of treatment with PPG extracts. Behavioral responses were scored according to the behavioral index described in the Materials and methods. Bars denote the median and whiskers the range of workers' behavioral responses. Groups tested after 2 and $15 \mathrm{~h}$ were analyzed separately using a Kruskal-Wallis-ANOVA with multiple comparisons. Asterisks indicate significant differences at a level of $P<0.05$, ns not significant

(pooled data, KW-test: $\mathrm{H}(1 ; 62)=8.80 ; P<0.01$, mc: $P<0.01)$.

The four groups of yoke-workers tested after $15 \mathrm{~h}$ of incubation also differed significantly in their behavioral responses towards encounter-workers (Fig. 2b, KW-test: $\mathrm{H}(3 ; 60)=13.11 ; P<0.01)$. In encounters with $\mathrm{NM}$ encounter-workers, yoke-workers showed low aggression, irrespective of yoke-worker-treatment (Fig. $2 \mathrm{~b}$ left, mc: $P>>0.05$ ). Interestingly, in encounters with n-NM encounter workers, $\mathrm{n}-\mathrm{NM}$ yoke-workers now (after $15 \mathrm{~h}$ of treatment) showed significantly lower aggression than NM yoke-workers (Fig. $1 \mathrm{~b}$ right, mc: $P<0.05$ ). The NM yokeworkers were still aggressive towards n-NM encounterworkers. They were significantly more aggressive towards $\mathrm{n}$-NM encounter-workers than both $\mathrm{NM}$ and $\mathrm{n}$-NM 


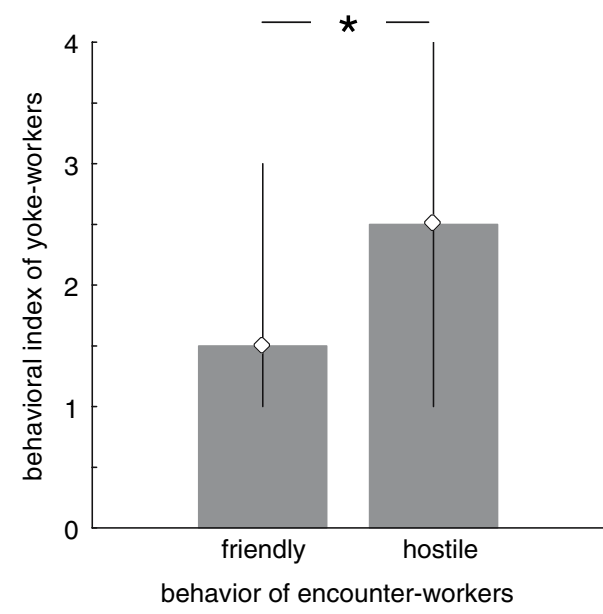

Fig. 3 The behavioral responses of encounter-workers (dichotomy data, friendly or hostile) compared to the behavioral responses of yokeworkers. Bars denote the median and whiskers the range of workers' behavioral responses. All 122 encounters were analyzed using a Spearmans rank correlation $(R=0.57 ; P<0.05)$

yoke-workers towards NM-encounter workers (Fig. 2b, mc: $P<0.05$ for both pairs).

The behavior shown by yoke-workers correlated with the behavior shown by encounter-workers (Fig. 3). Yokeworkers had higher behavioral index scores when encounter-workers showed aggression (hostile) than when they showed no aggression (friendly) (SR: $R=0.57 ; n=122$; $P<0.05)$. A significant correlation was found within five of the eight groups, and in all four of the groups tested after $15 \mathrm{~h}$ of yoke-worker-treatment (SR: $R=0.49$ to 0.63 ; $n=13$ to $17 ; P<0.05$ for all five groups, indicated by an asterisk in Table 1). Note that only a behavioral index of 3 and more clearly indicates aggression; thus a correlation between hostile encounter-workers and yoke-workers not necessarily indicates aggressive behavior of yoke-workers.

The behavioral difference found in yoke-workers might be influenced by the behavior of encounter-workers (Table 1). When comparing encounters with yoke-workers treated for $2 \mathrm{~h}$, NM encounter-workers showed less often aggressive behavior towards n-NM yoke-workers than nNM encounter-workers $\left(\mathrm{Chi}^{2}=5.01, P<0.05\right)$. Likewise, NM encounter-workers showed less often aggressive behavior towards NM yoke-workers than n-NM encounterworkers $\left(\mathrm{Chi}^{2}=7.80, P<0.01\right)$.

When comparing encounters with yoke-workers treated for $15 \mathrm{~h}$, aggression occurred less often in NM encounter-workers (in about $50 \%$ of the cases) than in n$\mathrm{NM}$ encounter-workers (in about $80 \%$ of the cases). We found no influence of the yoke-worker-treatment, neither on the behavioral response of NM encounter-workers $\left(\mathrm{Chi}^{2}=0.03, P=0.58\right)$ nor on the behavioral response of n-NM encounter-workers $\left(\mathrm{Chi}^{2}=0.04, P=0.44\right)$. Note that, in contrast, the behavioral response of yoke-workers in such encounters differed significantly (Fig. $2 b$ right).

For all pairs of independent groups, the encounter-workers' behavior corresponded to the behavior observed in yoke-workers, except for one pair: After a treatment of $15 \mathrm{~h}, \mathrm{n}$-NM yoke-workers showed low aggression scores towards n-NM encounter-workers but, in reverse, n-NM encounter-workers showed aggressive behavior towards NM- and n-NM yoke-workers equally often.

\section{Discussion}

Our study shows that the sensory experience of immobilized workers of the carpenter ant Camponotus floridanus can be manipulated with non-nestmate PPG extracts and that the manipulation later leads to a change in behavior. Tethered workers (yoke-workers) treated with non-nestmate PPG extract showed a reduction in aggressive behavior towards freely moving non-nestmates, after $15 \mathrm{~h}$ of antennal treatment. However, after $2 \mathrm{~h}$ of treatment, they showed a similar behavioral response towards encounterworkers than yoke-workers treated with nestmate PPG extract.

Table 1 Behavioral responses of encounter-workers towards yoke-workers

\begin{tabular}{|c|c|c|c|c|c|}
\hline & & \multicolumn{2}{|c|}{ NM encounter-worker } & \multicolumn{2}{|c|}{ n-NM encounter-worker } \\
\hline & & NM yoke-worker & n-NM yoke-worker & NM yoke-worker & n-NM yoke-worker \\
\hline \multirow[t]{3}{*}{$2 \mathrm{~h}$} & Friendly $(n)$ & 7 & 9 & 1 & 2 \\
\hline & Hostile $(n)$ & 7 & 8 & 16 & 12 \\
\hline & Total $(n)$ & 14 & $17 *$ & 17 & 14 \\
\hline \multirow[t]{3}{*}{$15 \mathrm{~h}$} & Friendly $(n)$ & 9 & 7 & 2 & 4 \\
\hline & Hostile (n) & 8 & 7 & 11 & 12 \\
\hline & Total $(n)$ & $17 *$ & $14^{*}$ & $13 *$ & $16^{*}$ \\
\hline
\end{tabular}

* Denote significant correlation between encounter-workers behavior and yoke-workers behavior (Spearmans-Rank correlation; $R=0.49$ to 0.63 ; $P<0.05)$ 
We used immobilized yoke-workers to prevent any interaction with other workers prior to behavioral testing and to provoke a change in the neuronal template only due to constant stimulation of the ants' antennae. Yoke-workers treated with PPG extracts displayed a low level of aggression towards encountering nestmates (median aggression scores of $\leq 2$, only scores $\geq 3$ clearly indicate aggression). Similarly low-aggression scores were measured with untreated yoke-workers encountering nestmates, in the pilot experiments that we used to establish the aggression index (data not shown). These results and the finding of specific aggression of yoke-workers towards non-nestmates indicate that the antennal treatment itself has little impact on the general discrimination ability of workers.

\section{Response to non-nestmates}

We found that yoke-workers were highly aggressive towards non-nestmates, after $2 \mathrm{~h}$ following the treatment with either PPG extract. After $15 \mathrm{~h}$, however, yoke-workers showed a different behavioral response towards non-nestmates. Their behavioral response depended on the kind of treatment they had received: Yoke-workers treated with non-nestmate PPG extracts showed a lower level of aggression than yoke-workers treated with nestmate PPG extract.

Is this change in behavior of yoke-workers treated with non-nestmate PPG extract caused by the prolonged antennal treatment or is it induced by a change in behavior of encounter-workers? We are confident that the observed change in aggressive behavior of yoke-workers was provoked by the antennal treatment and not by a change in behavior of encounter-workers because non-nestmate encounter-workers more often showed aggressive behavior than nestmate encounter-workers, irrespective of the treatment the yoke-workers received. In particular, non-nestmate encounter-workers often showed aggressive behavior towards yoke-workers treated with non-nestmate PPG extract for $15 \mathrm{~h}$ although these yoke-workers showed less often aggressive behavior than yoke-workers treated with nestmate PPG extract. This result seemingly contradicts the correlation found between the behavior of non-nestmate encounter-workers and non-nestmate yoke-workers after $15 \mathrm{~h}$ treatment (Fig. 2, Table 1, bottom right). Since only few of the yoke-workers in this group had a behavioral index of 3 or more (clearly aggressive behavior, 3 out of 16), the correlation probably describes a stronger but nonaggressive behavioral reaction of yoke-workers towards aggressive non-nestmate encounter-workers.

In addition to providing evidence for the importance of chemical cues, this result indicates that visual cues at most play a minor role in the nestmate recognition process of yoke-workers. However, it might well be that free running workers may use both, chemical and visual cues for nestmate recognition because perception of visual stimuli often requires self-generated optical flow (Hori et al. 2006).

Our results indicate a label-template matching of the non-nestmate label due to a reformation of the template in yoke-workers treated with n-NM PPG extract. Reformation of the template may start right after manipulation but it takes at least $2 \mathrm{~h}$ for functional adjustment of the template.

Given that ant CHC-profiles are not stable but influenced by environmental factors and that they vary with age, reproductive status, and caste membership of their bearers, it is expected that the neuronal template used for phenotype matching expresses plasticity to allow for a rapid behavioral change (Morel et al. 1988; Wagner et al. 1998; Nielsen et al. 1999; Heinze et al. 2002; Dietemann et al. 2003; D'Ettorre et al. 2004; Endler et al. 2004; Buczkowski et al. 2005; Katzerke et al. 2006). This template plasticity appears to primarily result in an extension of behavioral tolerance as has been demonstrated for several ant species where individuals of one species expressed a broader acceptance range towards individuals of a different species when they had been reared in mixed species groups (Errard et al. 2006). Errard et al. showed that greater dissimilarity in the CHC-profiles of workers in mixed species groups resulted in a broader acceptance range based on a broader template rather than on multiple templates.

\section{Response to nestmates}

Yoke-workers treated with either PPG extract showed no different behavioral response towards nestmates, neither after $2 \mathrm{~h}$ nor after $15 \mathrm{~h}$ of treatment. Thus, the acceptance range of manipulated workers is broadened, after $15 \mathrm{~h}$ of non-nestmate treatment, with phenotype matching to the nestmate label as well as to the non-nestmate label. It remains to be examined whether, on a longer time scale, template reformation will lead to further adjustment by constriction of the template's acceptance range (fine-tuned template) to the sensory experience, which, in our experiment, is represented by the non-nestmate label. In honeybees, the corporate fostering of workers from different, unrelated colonies also leads to a change in template (Harano and Sasaki 2006). Harano and Sasaki also found that it takes more than a day of exposure to non-nestmates before workers start to respond aggressively towards their former nestmates indicating a lagged fine-tuning.

Temporal dynamic of template reformation

It is expected that the reformation of the template is adapted to the temporal structure of naturally occurring changes in the CHC-profiles. In honeybees, the template reformation occurs within $25 \mathrm{~min}$ of exposure (Harano and Sasaki 2006), whereas in our study it took more than $2 \mathrm{~h}$. This is a 
much faster adjustment. Possibly, the flower-scent loaded nectar collected by honeybees cause faster changes in the CHC-profile of the colony than the food collected by $C$. floridanus. Unlike to our experiment, in other experiments investigating the template reformation of ants and bees, individuals were allowed to mutually and frequently interact with non-nestmates. They were able to exchange CHCs (Vienne et al. 1995) and to frequently resample the CHCprofiles of nearby non-nestmate workers. The influence of repeated interaction and sampling of the non-nestmate workers' CHC-profile may influence the process of template reformation which remains to be investigated.

The transient time for a change in the colony CHC-profile results in a larger variation of worker CHC-profiles within a colony. To account for such variation, template reformation is expected to result in a broadened acceptance range of workers which is supported by our and others' results. The template reformation process seems to consist of this initial phase, and a delayed phase in which the template is finetuned. These two phases require a neuronal plasticity which hardly can be implemented at the level of the olfactory receptor neurons, even if many of them are assembled as a functional unit in one sensillum. Recently, Ozaki et al. proposed that the sensory response at a chemosensory sensillum may function as template (Ozaki et al. 2005). The authors suggested a specific adaptation of the response of multiple receptor neurons to nestmate CHC-profiles as a possible mechanism. Even if the proposed mechanism of a complex adaptation to ratios of multiple components exists, it cannot explain the two phases of template reformation process described above yet. For insect olfactory receptor neurons, several different adaptation mechanisms were described (Kurahashi and Menini 1997; Redkozubov 2000; Dolzer et al. 2003). However, all of them are acting on a shorter time scale as the behavioral change described in this study $(>2 \mathrm{~h}$ ) and, presumably, even on a shorter time frame as the faster template reformation process $(25 \mathrm{~min}$ ) found in honeybees (Vareschi 1971; Bittel and Martin 1974; Kaissling et al. 1987; Harano and Sasaki 2006). On a longer time scale, neuromodulators (e.g. octopamin and serotonin) may modify the sensory response as has been shown for the circadian change of olfactory receptor neuron sensitivity (Pophof 2000; Dolzer etal. 2001; Flecke et al. 2006). Neuromodulation acting on olfactory receptor neurons represents a global adjustment of sensitivity. Nevertheless, it is also unlikely to be a mechanism for such specific template formation as described in ants and honeybees. The slow reformation process of the template and its fine-tuned adjustment suggest CNS-plasticity rather than ORN-adaptation as a mechanism for template formation. However, further physiological studies on ORNs (EAG or single unit recordings) are necessary to investigate possible influences of sensory adaptation on nestmate recognition.
Our finding supports the hypothesis that plasticity of the central nervous system adjusts for changes in the CHC-profile of the colony, with aggression being regulated by a threshold of similarity between template and label (Crozier and Dix 1979; Obin and Vander Meer 1989; Vander Meer and Morel 1998).

Our results show that direct experimental manipulation of the workers' sensory experience can change the phenotype matching of their template. This technique can be used as a tool to study the template reformation process not only in behavioral experiments but also in physiological experiments investigating the representation of CHC-profiles in the ant's brain.

Acknowledgments We thank Annett Endler for collecting and taking care of the ant colonies that we used as well as for constructive comments on the experimental design as well as on this manuscript. We thank Linda S. Kuebler and Christina Kelber for helping with the experiments and Annette Laudahn for advice in rearing the colony. This study was funded by DFG, SFB 554/A6. The experiments comply with the "Principles of animal care", publication No. 86-23, revised 1985 of the National Institute of Health, and also with the current laws of Germany.

\section{References}

Akino T, Yamaoka R (2005) Trail discrimination signal of Lasius japonicus (Hymenoptera : Formicidae). Chemoecol 15(1):21-30

Bagneres AG, Morgan ED (1991) The postpharyngeal glands and the cuticle of Formicidae contain the same characteristic hydrocarbons. Experientia 47(1):106-111

Bhatkar AP, Whitcomb WH (1970) Artificial diet for rearing various species of ants. Fla Entomol 53:229-232

Bittel H, Martin H (1974) Olfactory fatigue in honeybee. J Comp Physiol A 89(3):293-311

Boulay R, Katzav-Gozansky T, Hefetz A, Lenoir A (2004) Odour convergence and tolerance between nestmates through trophallaxis and grooming in the ant Camponotus fellah (Dalla Torre). Insect Soc 51(1):55-61

Buczkowski G, Kumar R, Suib SL, Silverman J (2005) Diet-related modification of cuticular hydrocarbon profiles of the Argentine ant, Linepithema humile, diminishes intercolony aggression. J Chem Ecol 31(4):829-843

Crozier RH, Dix MW (1979) Analysis of 2 genetic models for the innate components of colony odor in social Hymenoptera. Behav Ecol Sociobiol 4(3):217-224

Crozier RH, Pamilo P (1996) Evolution of social insect colonies. Oxford University Press, Oxford

D'Ettorre P, Heinze E, Schulz C, Francke W, Ayasse M (2004) Does she smell like a queen? Chemoreception of a cuticular hydrocarbon signal in the ant Pachycondyla inversa. J Exp Biol 207(7):1085-1091

Dahbi A, Hefetz A, Cerda X, Lenoir A (1999) Trophallaxis mediates uniformity of colony odor in Cataglyphis iberica ants (Hymenoptera, Formicidae). J Insect Behav 12(4):559-567

Dietemann V, Peeters C, Liebig J, Thivet V, Holldobler B (2003) Cuticular hydrocarbons mediate discrimination of reproductives and nonreproductives in the ant Myrmecia gulosa. Proc Natl Acad Sci USA 100(18):10341-10346

Dolzer J, Krannich S, Fischer K, Steng1 M (2001) Oscillations of the transepithelial potential of moth olfactory sensilla are influenced by octopamine and serotonin. J Exp Biol 204(16):2781-2794 
Dolzer J, Fischer K, Stengl M (2003) Adaptation in pheromone-sensitive trichoid sensilla of the hawkmoth Manduca sexta. J Exp Biol 206(9):1575-1588

Endler A, Liebig J, Schmitt T, Parker JE, Jones GR, Schreier P, Holldobler B (2004) Surface hydrocarbons of queen eggs regulate worker reproduction in a social insect. Proc Natl Acad Sci USA 101(9):2945-2950

Errard C, Hefetz A, Jaisson P (2006) Social discrimination tuning in ants: template formation and chemical similarity. Behav Ecol Sociobiol 59(3):353-363

Flecke C, Dolzer J, Krannich S, Stengl M (2006) Perfusion with cGMP analogue adapts the action potential response of pheromone-sensitive sensilla trichoidea of the hawkmoth Manduca sexta in a daytime-dependent manner. J Exp Biol 209(19):3898-3912

Getz WM, Chapman RF (1987) An odor discrimination model with application to kin recognition in social insects. Int $\mathrm{J}$ Neurosci 32(3-4):963-978

Harano K, Sasaki M (2006) Renewal process of nestmate recognition template in European honeybee Apis mellifera L. (Hymenoptera : Apidae). Appl Entomol Zool 41(2):325-330

Hauber ME, Sherman PW (2001) Self-referent phenotype matching: theoretical considerations and empirical evidence. Trends Neurosci 24:609-616

Heinze J, Stengl B, Sledge MF (2002) Worker rank, reproductive status and cuticular hydrocarbon signature in the ant, Pachycondyla cf. inversa. Behav Ecol Sociobiol 52(1):59-65

Hölldobler B (1995) The chemistry of social regulation-multicomponent signals in ant societies. Proc Natl Acad Sci USA 92(1):19-22

Hölldobler B, Wilson EO (1990) The ants. Harvard University Press, Cambridge

Hori S, Takeuchi H, Arikawa K, Kinoshita M, Ichikawa N, Sasaki M, Kubo T (2006) Associative visual learning, color discrimination, and chromatic adaptation in the harnessed honeybee Apis mellifera L. J Comp Physiol A 192(7):691-700

Howard RW (1993) Cuticular hydrocarbons and chemical communication. In: Stanley-Hammelson DW, Nelson DR (eds) Insect lipids: chemistry, biodiversity and biology. University of Nebraska, Nebraska, pp 179-226

Kaissling K-E, Zack-Strausfeld C, Rumbo E (1987) Adaptation of processes in insect olfactory receptors: mechanisms and behavioral significance. In: Roper SD, Atema J (eds) New York Academy of Sciences, Colorado, pp 104-112

Kandel ER, Wurtz RH (1990) Constructing the visual image. In: Kandel ER, Schwartz JH, Jessell TM (eds) Principles of neural science, 4th edn. McGraw-Hill, New York, pp 492-506

Katzerke A, Neumann P, Pirk CWW, Bliss P, Moritz RFA (2006) Seasonal nestmate recognition in the ant Formica exsecta. Behav Ecol Sociobiol 61(1):143-150

Kurahashi T, Menini A (1997) Mechanism of odorant adaptation in the olfactory receptor cell. Nature 385(6618):725-729

Lacy RC, Sherman PW (1983) Kin recognition by phenotype matching. Am Nat 121(4):489-512

Lahav S, Soroker V, Hefetz A, Vander Meer RK (1999) Direct behavioral evidence for hydrocarbons as ant recognition discriminators. Naturwissenschaften 86(5):246-249
Lenoir A, Fresneau D, Errard C, Hefetz A (1999) Individuality and colonial identity in ants: the emergence of the social representation concept. In: Detrain C, Deneubourg JL, Pasteels JM (eds) Information processing in social insects. Birkhäuser Verlag, Berlin, pp 219-237

Lenoir A, Hefetz A, Simon T, Soroker V (2001) Comparative dynamics of gestalt odour formation in two ant species Camponotus fellah and Aphaenogaster senilis (Hymenoptera : Formicidae). Physiol Entomol 26(3):275-283

Lockey KH (1988) Lipids of the insect cuticle: origin, composition and function. Comp Biochem Physiol B 89B:595-645

Lucas C, Pho DB, Jallon JM, Fresneau D (2005) Role of cuticular hydrocarbons in the chemical recognition between ant species in the Pachycondyla villosa species complex. J Insect Physiol 51(10):1148-1157

Morel L, Vandermeer RK, Lavine BK (1988) Ontogeny of nestmate recognition cues in the red carpenter ant (Camponotus floridanus) -behavioral and chemical evidence for the role of age and social experience. Behav Ecol Sociobiol 22(3):175-183

Nielsen J, Boomsma JJ, Oldham NJ, Petersen HC, Morgan ED (1999) Colony-level and season-specific variation in cuticular hydrocarbon profiles of individual workers in the ant Formica truncorum. Insect Soc 46(1):58-65

Obin MS, Vander Meer RK (1989) Mechanism of template-label matching in fire ant, Solenopsis invicta Buren, nestmate recognition. Anim Behav 38:430-435

Ozaki M, Wada-Katsumata A, Fujikawa K, Iwasaki M, Yokohari F, Satoji Y, Nisimura T, Yamaoka R (2005) Ant nestmate and nonnestmate discrimination by a chemosensory sensillum. Science 309(5732):311-314

Pophof B (2000) Octopamine modulates the sensitivity of silkmoth pheromone receptor neurons. J Comp Physiol A 186:307-313

Redkozubov A (2000) Guanosine 3',5' -cyclic monophosphate reduces the response of the Moth's olfactory receptor neuron to pheromone. Chem Senses 25(4):381-385

Singer TL (1998) Roles of hydrocarbons in the recognition systems of insects. Am Zool 38(2):394-405

Soroker V, Vienne C, Hefetz A, Nowbahari E (1994) The postpharyngeal gland as a Gestalt organ for nestmate recognition in the ant Cataglyphis niger. Naturwissenschaften 81(11):510-513

Vander Meer RK, Morel L (1998) Nestmate recognition in ants. In: Vander Meer RK, Winston M, Espelie KE (eds) Pheromone communication in social insects. Westview, Boulder, pp 79-103

Vareschi E (1971) Odor discrimination in honey bee-single cell and behavioral response. Z vergl Physiol 75(2):143-173

Vienne C, Soroker V, Hefetz A (1995) Congruency of hydrocarbon patterns in heterospecific groups of ants-transfer and/or biosynthesis. Insect Soc 42(3):267-277

von Ehrenfels C (1890) Über Gestaltqualitäten. Vierteljahrsschrift für wissenschaftliche Philosophie 14:249-292

Wagner D, Brown MJF, Broun P, Cuevas W, Moses LE, Chao DL, Gordon DM (1998) Task-related differences in the cuticular hydrocarbon composition of harvester ants, Pogonomyrmex barbatus. J Chem Ecol 24(12):2021-2037

Wertheimer M (1925) Über Gestalttheorie. Philosophische Zeitschrift für Forschung und Aussprache 1:39-60 